\title{
Asymptotic Behavior of the Bifurcation Diagrams for Semilinear Problems with Application to Inverse Bifurcation Problems
}

\author{
Tetsutaro Shibata \\ Laboratory of Mathematics, Institute of Engineering, Hiroshima University, Higashihiroshima 739-8527, Japan \\ Correspondence should be addressed to Tetsutaro Shibata; shibata@amath.hiroshima-u.ac.jp
}

Received 22 October 2014; Accepted 15 December 2014

Academic Editor: Nasser-Eddine Tatar

Copyright (C) 2015 Tetsutaro Shibata. This is an open access article distributed under the Creative Commons Attribution License, which permits unrestricted use, distribution, and reproduction in any medium, provided the original work is properly cited.

We consider the nonlinear eigenvalue problem $u^{\prime \prime}(t)+\lambda f(u(t))=0, u(t)>0, t \in I=:(-1,1), u(1)=u(-1)=0$, where $f(u)$ is a cubic-like nonlinear term and $\lambda>0$ is a parameter. It is known by Korman et al. (2005) that, under the suitable conditions on $f(u)$, there exist exactly three bifurcation branches $\lambda=\lambda_{j}(\xi)(j=1,2,3)$, and these curves are parameterized by the maximum norm $\xi$ of the solution $u_{\lambda}$ corresponding to $\lambda$. In this paper, we establish the precise global structures for $\lambda_{j}(\xi)(j=1,2,3)$, which can be applied to the inverse bifurcation problems. The precise local structures for $\lambda_{j}(\xi)(j=1,2,3)$ are also discussed. Furthermore, we establish the asymptotic shape of the spike layer solution $u_{2}(\lambda, t)$, which corresponds to $\lambda=\lambda_{2}(\xi)$, as $\lambda \rightarrow \infty$.

\section{Introduction}

We consider the following nonlinear eigenvalue problem:

$$
\begin{gathered}
u^{\prime \prime}(t)+\lambda f(u(t))=0, \quad t \in I=:(-1,1), \\
u(t)>0, \quad t \in I, \\
u(1)=u(-1)=0,
\end{gathered}
$$

where $f(u)$ is a cubic-like nonlinear term and $\lambda>0$ is a parameter. We assume the following conditions (A.1)-(A.3), which have been introduced in [1].

(A.1) $f(u)$ is a $C^{2}$-function on $[0, \infty)$ and has three positive roots at $0<a<b<c$, and

$$
\begin{gathered}
f(u)>0 \quad \text { on }[0, a) \cup(b, c), \\
f(u)<0 \quad \text { on }(a, b) \cup(c, \infty), \\
\int_{a}^{c} f(s) d s>0 .
\end{gathered}
$$

(A.2) There exists a constant $a<\beta<c$ such that

$$
\begin{gathered}
f^{\prime \prime}(u)>0 \quad \text { for } 0 \leq u<\beta, \\
f^{\prime \prime}(u)<0 \quad \text { for } u>\beta .
\end{gathered}
$$

(A.3) Let $\tau \in(b, c)$ satisfy $f^{\prime}(\tau)=0$. Then

$$
\int_{a}^{\tau} f(s) d s \leq 0
$$

The typical example of $f(u)$ which satisfies (A.1)-(A.3) is $f(u)=(u-a)(u-b)(c-u)$ with $c>2 b-a$ and the area of the negative hump of $f(u)$ is nearly equal to that of the positive hump. For example, if we choose $b<(a+c) / 2$ appropriately, then $f(u)=(u-a)(u-b)(c-u)$ satisfies (A.1)-(A.3).

Nonlinear elliptic eigenvalue problems have been studied by many authors. We refer to [2-8] and the references therein. Among other things, (1)-(3) have been investigated by many authors. We refer to $[1,9-12]$ and the references therein. In particular, the following basic properties of the structure of bifurcation diagram for (1)-(3) have been proved in [1, Theorem 3.1].

Theorem 1 (see [1, Theorem 3.1]). Assume (A.1)-(A.3). Then there exists a critical $\lambda_{0}$ such that (1)-(3) have exactly one positive solution for $0<\lambda<\lambda_{0}$, exactly two positive solutions for $\lambda=\lambda_{0}$, and exactly three positive solutions for $\lambda>\lambda_{0}$. Furthermore, all solutions lie on two smooth solution curves, and $\lambda$ is parameterized by $\xi:=\left\|u_{\lambda}\right\|_{\infty}$ as $\lambda=\lambda(\xi)$. One of the 


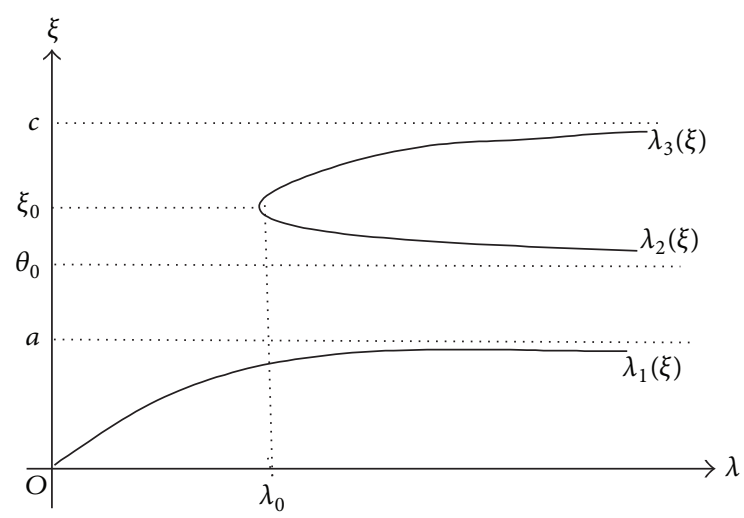

FIgURe 1

curves, referred to as the lower curve $\lambda_{1}(\xi)$, satisfies $\lambda_{1}(0)=0$ and increases in $0 \leq \xi<a$, and

$$
\begin{gathered}
\lambda_{1}(\xi) \longrightarrow \infty \quad \text { as } \xi \longrightarrow a, \\
u_{1}(\lambda, t) \longrightarrow a \text { for } t \in I \text { as } \lambda \longrightarrow \infty,
\end{gathered}
$$

where $u_{1}(\lambda, t)$ is a solution of (1)-(3) corresponding to $\lambda=$ $\lambda_{1}(\xi)$. (Note that $\lambda \rightarrow \infty$ is equivalent to $\xi \rightarrow$ a.) The upper curve, which consists of two branches $\lambda_{j}(\xi)(j=2,3)$, is a parabola-like curve with exactly one turn to the right at $\xi=\xi_{0} \in(b, c)$. Furthermore,

$$
\begin{gathered}
\lambda_{2}(\xi) \longrightarrow \infty \quad \text { as } \xi \longrightarrow \theta_{0}, \\
u_{2}(\lambda, 0) \longrightarrow \theta_{0}, \quad u_{2}(\lambda, t) \longrightarrow a \\
(t \in I \backslash\{0\}) \quad \text { as } \lambda \longrightarrow \infty, \\
\lambda_{3}(\xi) \longrightarrow \infty \quad \text { as } \xi \longrightarrow c, \\
u_{3}(\lambda, t) \longrightarrow c \quad(t \in I) \text { as } \lambda \longrightarrow \infty,
\end{gathered}
$$

where $u_{j}(\lambda, t)$ is a solution of (1)-(3) corresponding to $\lambda=$ $\lambda_{j}(\xi)(j=2,3)$ and $\theta_{0} \in(b, c)$ is a constant which satisfies

$$
\int_{a}^{\theta_{0}} f(s) d s=0
$$

(See Figure 1 for the bifurcation diagram.)

As we see from (9), (11), and (13), one of the most interesting facts in Theorem 1 is the difference between the asymptotic shapes of $u_{j}(\lambda, t)(j=1,3)$ and $u_{2}(\lambda, t)$ for $\lambda$ sufficiently large (see Figures 2 and 3 ). This difference comes from the fact that $u_{j}(\lambda, t)(j=1,3)$ is stable and $u_{2}(\lambda, t)$ is unstable. This drives us to the question whether these facts give effect on the asymptotic behavior of the bifurcation branches or not.

The purpose of this paper is to establish the precise asymptotic formulas for $\lambda_{j}(\xi)(j=1,2,3)$ as $\xi \rightarrow a, \theta_{0}, c$, respectively, to clarify how the difference of the asymptotic shapes of solutions corresponding to these three curves gives effect to the asymptotic formulas for $\lambda_{j}(\xi)(j=1,3)$ and $\lambda_{2}(\xi)$. Furthermore, we establish the asymptotic widths $t_{a, \lambda}$ and $t_{d, \lambda}$ of the spike layer of solution $u_{2}(\lambda, t)$ as $\xi \rightarrow \theta_{0}$.

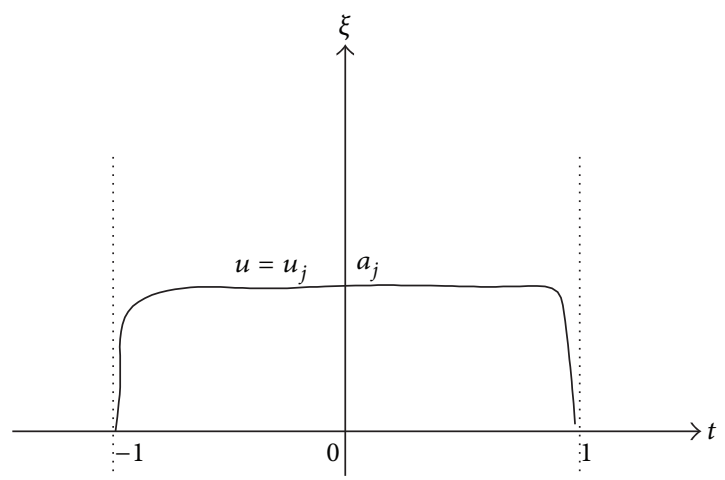

FIGURE 2

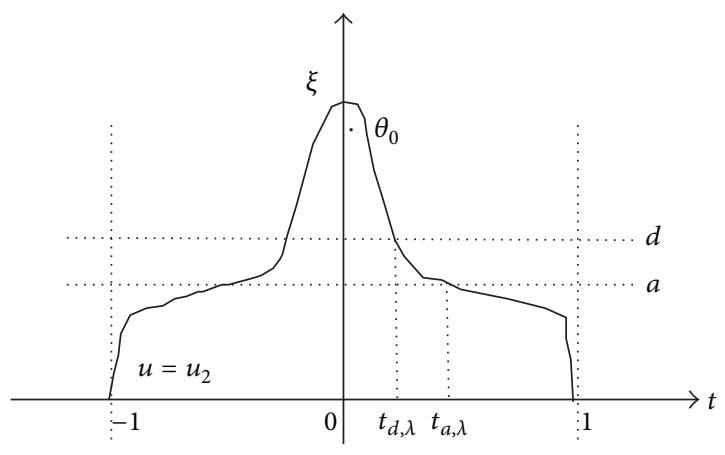

Figure 3

Finally, we establish the precise asymptotic formulas for $\lambda_{1}(\xi)$ as $\xi \rightarrow 0$ and $\lambda_{j}(\xi)$ as $\xi \rightarrow \xi_{0}(j=2,3)$. By using the asymptotic formulas above, we propose new inverse bifurcation problems.

Now we state our main result. Let $F(u):=\int_{0}^{u} f(s) d s$.

Theorem 2. Assume that $0<\xi<$ a. Let $\delta_{\xi}>0$ satisfy $\log \delta_{\xi}=$ $\delta_{\xi} \log (a-\xi)$. Then as $\xi \rightarrow a$,

$$
\lambda_{1}(\xi)=\frac{1}{\left|f^{\prime}(a)\right|}\left(-\log (a-\xi)+O\left(\log \delta_{\xi}\right)\right)^{2} .
$$

Theorem 3. Assume that $\xi>\theta_{0}$. Let $\delta_{\xi}>0$ satisfy $\log \delta_{\xi}=$ $\delta_{\xi} \log \left(\xi-\theta_{0}\right)$.

(i) $A s \xi \rightarrow \theta_{0}$,

$$
\lambda_{2}(\xi)=\frac{1}{\left|f^{\prime}(a)\right|}\left(-\log \left(\xi-\theta_{0}\right)+O\left(\log \delta_{\xi}\right)\right)^{2} .
$$

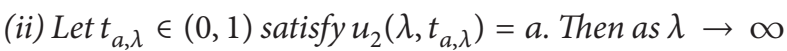
(namely, $\xi \rightarrow \theta_{0}$ ),

$$
t_{a, \lambda}=\frac{1}{2}+O\left(\frac{\left(\xi-\theta_{0}\right)^{1 / 2}}{\log \left(\xi-\theta_{0}\right)}\right) .
$$

(iii) Let $d \in\left(a, \theta_{0}\right)$ be an arbitrary fixed constant. Furthermore, let $t_{d, \lambda} \in(0,1)$ satisfy $u_{2}\left(\lambda, t_{d, \lambda}\right)=d$. Then as $\lambda \rightarrow \infty$ (namely, $\left.\xi \rightarrow \theta_{0}\right)$,

$$
t_{d, \lambda}=\frac{1}{\sqrt{2 \lambda}}\left(C_{d}+O\left(\left(\xi-\theta_{0}\right)^{1 / 2}\right)\right),
$$


where

$$
C_{d}:=\int_{d}^{\theta_{0}} \frac{1}{\sqrt{F\left(\theta_{0}\right)-F(\theta)}} d \theta
$$

Theorem 4. Assume that $\xi<c$. Let $\delta_{\xi}>0$ satisfy $\log \delta_{\xi}=$ $\delta_{\xi} \log (c-\xi)$. Then as $\xi \rightarrow c$,

$$
\lambda_{3}(\xi)=\frac{1}{\left|f^{\prime}(c)\right|}\left(-\log (c-\xi)+O\left(\log \delta_{\xi}\right)\right)^{2} .
$$

Now, we establish the local asymptotic behavior of $\lambda_{1}(\xi)$ and $\lambda_{j}(\xi)(j=2,3)$ near $\xi=0$ and $\xi=\xi_{0}$, respectively, where $\xi_{0} \in\left(\theta_{0}, 1\right)$ satisfy

$$
\begin{aligned}
& 2 \int_{0}^{1} \frac{1}{\sqrt{F\left(\xi_{0}\right)-F\left(\xi_{0} s\right)}} d s \\
& \quad=\xi_{0} \int_{0}^{1}\left(F\left(\xi_{0}\right)-F\left(\xi_{0} s\right)\right)^{-3 / 2}\left(f\left(\xi_{0}\right)-s f\left(\xi_{0} s\right)\right) d s .
\end{aligned}
$$

Theorem 5. (i) As $\xi \rightarrow 0$,

$$
\lambda_{1}(\xi)=\frac{\xi}{2 f(\xi)}\left(2+\sum_{k=1}^{\infty} L_{k}(\xi) \xi^{k}\right)^{2},
$$

where $\left\{L_{k}(\xi)\right\}(k=1,2, \ldots)$ are bounded functions of $\xi$ determined explicitly.

(ii) Assume that $f(u)$ is analytic near $u=0$. Then as $\xi \rightarrow$ 0 ,

$$
\lambda_{1}(\xi)=\frac{2}{f(0)} \xi+\sum_{k=1}^{\infty} M_{k}(\xi) \xi^{k}
$$

where $\left\{M_{k}(\xi)\right\}(k=1,2, \ldots)$ are bounded functions of $\xi$ determined explicitly.

Theorem 6. Let $j=2,3$. Then as $\xi \rightarrow \xi_{0}$,

$$
\lambda_{j}(\xi)=\lambda_{0}+\sum_{k=1}^{\infty} J_{k}(\xi)\left(\xi-\xi_{0}\right)^{k}
$$

where $\left\{J_{k}(\xi)\right\}(k=1,2, \ldots)$ are bounded functions determined explicitly.

Finally, we apply our results above to the inverse bifurcation problems.

Theorem 7. Let $k=1,2$. Assume that $f_{k}(u)=\left(u-a_{k}\right)(u-$ $\left.b_{k}\right)\left(c_{k}-u\right)$ satisfy (A.1)-(A.3), where $0<a_{k}<b_{k}<c_{k}$ are unknown constants. Let $\lambda_{j}(k, \xi)(j=1,3)$ be the bifurcation branches corresponding to $f_{k}(u)$. Further, let $\theta_{k, 0}$ be the number defined in (14) for $f_{k}(u)$. Finally, for $\eta:=a_{1}-\xi_{1}=a_{2}-\xi_{2}>0$ and $\zeta:=c_{1}-\xi_{1}=c_{2}-\xi_{2}>0$, let

$$
\mu_{1}(k, \eta):=\lambda_{1}\left(k, \xi_{k}\right), \quad \mu_{3}(k, \zeta):=\lambda_{3}\left(k, \xi_{k}\right)
$$

(i) Suppose that

$$
\begin{gathered}
\lim _{\eta \rightarrow 0} \frac{\mu_{1}(1, \eta)}{\mu_{1}(2, \eta)}=1, \\
\lambda_{1}(1, \xi)-\lambda_{1}(2, \xi)=o\left(\xi^{2}\right) \quad \text { as } \xi \longrightarrow 0 .
\end{gathered}
$$

Then, $a_{1}=a_{2}, b_{1}=b_{2}$, and $c_{1}=c_{2}$.

(ii) Suppose that

$$
\begin{gathered}
\lim _{\eta \rightarrow 0} \frac{\mu_{1}(1, \eta)}{\mu_{1}(2, \eta)}=\lim _{\zeta \rightarrow 0} \frac{\mu_{3}(1, \zeta)}{\mu_{3}(2, \zeta)}=1, \\
\lambda_{1}(1, \xi)-\lambda_{1}(2, \xi)=o(\xi) \text { as } \xi \longrightarrow 0 .
\end{gathered}
$$

Then, $a_{1}=a_{2}, b_{1}=b_{2}$, and $c_{1}=c_{2}$.

(iii) Suppose that (28) and $\theta_{1,0}=\theta_{2,0}$ hold. Then, $a_{1}=a_{2}$, $b_{1}=b_{2}$, and $c_{1}=c_{2}$.

Theorem 7(i) implies that if we assume that the unknown nonlinear term $f(u)$ is a cubic, then the precise information about the local asymptotic behavior along with the rough global asymptotics of one branch determines the unknown $f(u)$. Theorem 7(ii) implies that the unknown $f(u)$ is determined by only the asymptotic behavior of two branches.

Our methods to prove Theorems 2-4 are based on the precise calculation of the time map. We prove Theorems 5 and 6 by the method developed in [13]. By using Theorems 2, 4, and 5, we prove Theorem 7 .

\section{Proof of Theorem 2}

In this section, let $\xi<a$ and let $\xi \rightarrow a$. In what follows, $C$ denotes various positive constants independent of $\lambda \gg 1$. We know that if $(\lambda, u) \in \mathbb{R}_{+} \times C^{2}(\bar{I})$ satisfies (1)-(3), then

$$
\begin{gathered}
u(t)=u(-t), \quad 0 \leq t \leq 1, \\
u(0)=\max _{-1 \leq t \leq 1} u(t), \\
u^{\prime}(t)<0, \quad 0<t \leq 1 .
\end{gathered}
$$

We parameterize the solution pair by using the $L^{\infty}$ norm of the solution $\xi=\left\|u_{\lambda}\right\|_{\infty}$ such as $(\lambda, u)=\left(\lambda_{1}(\xi), u_{\xi}\right)(0 \leq \xi<$ a). By (1), for $t \in \bar{I}$,

$$
\left[u_{\xi}^{\prime \prime}(t)+\lambda f\left(u_{\xi}(t)\right)\right] u_{\xi}^{\prime}(t)=0 .
$$

This implies that, for $t \in \bar{I}$,

$$
\frac{d}{d t}\left[\frac{1}{2} u_{\xi}^{\prime}(t)^{2}+\lambda F\left(u_{\xi}(t)\right)\right]=0
$$

By this, (31), and putting $t=0$, for $-1 \leq t \leq 1$, we obtain

$$
u_{\xi}^{\prime}(t)^{2}+2 \lambda F\left(u_{\xi}(t)\right)=\text { constant }=2 \lambda F(\xi) .
$$

This implies that

$$
u_{\xi}^{\prime}(t)^{2}=2 \lambda\left(F(\xi)-F\left(u_{\xi}(t)\right)\right) .
$$


By this and (32), for $0 \leq t \leq 1$, we obtain

$$
-u_{\xi}^{\prime}(t)=\sqrt{2 \lambda\left(F(\xi)-F\left(u_{\xi}(t)\right)\right)} .
$$

By this, for $0 \leq t \leq 1$,

$$
\begin{aligned}
\sqrt{\lambda} & =\frac{1}{\sqrt{2}} \int_{0}^{1} \frac{-u_{\xi}^{\prime}(t)}{\sqrt{F(\xi)-F\left(u_{\xi}(t)\right)}} d t \\
& =\frac{1}{\sqrt{2}} \int_{0}^{\xi} \frac{1}{\sqrt{F(\xi)-F(\theta)}} d \theta .
\end{aligned}
$$

By mean value theorem, for $0<\theta<\xi$, we have

$$
F(\xi)-F(\theta)=f(\xi)(\xi-\theta)-\frac{1}{2} f^{\prime}\left(\xi_{\theta}\right)(\xi-\theta)^{2},
$$

where $\theta<\xi_{\theta}<\xi$. We know that $f^{\prime}\left(\xi_{\theta}\right)<0$, since, by (A.2), $f^{\prime}(0)<f^{\prime}(y)<f^{\prime}(a)<0$ for $0<y<a$. Let $\delta:=\delta_{\xi}>0$ defined in Theorem 2 . We easily see that $\delta_{\xi} \rightarrow 0$ as $\xi \rightarrow a$. Furthermore, by Taylor expansion, as $\xi \rightarrow a$,

$$
\begin{gathered}
f(\xi)=f^{\prime}(a)(\xi-a)+O\left((\xi-a)^{2}\right), \\
a-\xi=\delta_{\xi}^{1 / \delta_{\xi}} \ll \delta_{\xi} .
\end{gathered}
$$

By (38) and (39), we obtain

$$
\begin{aligned}
\sqrt{\lambda}= & \frac{1}{\sqrt{2}} \int_{0}^{\xi} \frac{1}{\sqrt{f(\xi)(\xi-\theta)-f^{\prime}\left(\xi_{\theta}\right)(\xi-\theta)^{2} / 2}} d \theta \\
= & \frac{1}{\sqrt{2}}\left\{\int_{\xi-\delta}^{\xi} \frac{1}{\sqrt{f(\xi)(\xi-\theta)-f^{\prime}\left(\xi_{\theta}\right)(\xi-\theta)^{2} / 2}} d \theta\right. \\
& \left.+\int_{0}^{\xi-\delta} \frac{1}{\sqrt{f(\xi)(\xi-\theta)-f^{\prime}\left(\xi_{\theta}\right)(\xi-\theta)^{2} / 2}} d \theta\right\} \\
:= & \frac{1}{\sqrt{2}}\left(Z_{1}+Z_{2}\right) .
\end{aligned}
$$

We write $\xi(t):=\xi_{\xi-t}(0<t<\delta)$. Then,

$$
\begin{aligned}
Z_{1}= & \int_{0}^{\delta} \frac{1}{\sqrt{f(\xi) t+\left|f^{\prime}(\xi)\right| t^{2} / 2}} d t \\
& +\left(\int_{0}^{\delta} \frac{1}{\sqrt{f(\xi) t+\left|f^{\prime}(\xi(t))\right| t^{2} / 2}} d t\right. \\
& \left.\quad-\int_{0}^{\delta} \frac{1}{\sqrt{f(\xi) t+\left|f^{\prime}(\xi)\right| t^{2} / 2}} d t\right) \\
:= & Z_{11}+Z_{12} .
\end{aligned}
$$

Then,

$$
\begin{aligned}
& Z_{11}=\left[\sqrt{\frac{2}{\left|f^{\prime}(\xi)\right|}} \log || f^{\prime}(\xi) \mid t+f(\xi)\right. \\
&\left.+\sqrt{\left|f^{\prime}(\xi)\right|^{2} t^{2}+2\left|f^{\prime}(\xi)\right| f(\xi) t} \mid\right]_{t=0}^{t=\delta} \\
&= \sqrt{\frac{2}{\left|f^{\prime}(\xi)\right|}}(-\log f(\xi)+D)
\end{aligned}
$$

where

$$
\begin{aligned}
D & :=\log || f^{\prime}(\xi)\left|\delta+f(\xi)+\sqrt{\left|f^{\prime}(\xi)\right|^{2} \delta^{2}+2\left|f^{\prime}(\xi)\right| f(\xi) \delta}\right| \\
& =O(\log \delta)
\end{aligned}
$$

by $(40)$. We put

$$
\begin{gathered}
X:=\sqrt{f(\xi) t+\frac{1}{2}\left|f^{\prime}(\xi)\right| t^{2}}, \\
Y:=\sqrt{f(\xi) t+\frac{1}{2}\left|f^{\prime}(\xi(t))\right| t^{2}} .
\end{gathered}
$$

We note that $\xi-\delta<\xi(t)<\xi$. Then by (41) and (43),

$$
\begin{aligned}
\left|Z_{12}\right| & =\left|\frac{1}{2} \int_{0}^{\delta} \frac{\left(f^{\prime}(\xi)-f^{\prime}(\xi(t))\right) t^{2}}{X Y(X+Y)} d t\right| \\
& \leq C \int_{0}^{\delta} \frac{\left|f^{\prime \prime}(\xi)\right|(\xi-\xi(t))}{X} d t \\
& \leq C \delta \int_{0}^{\delta} \frac{1}{\sqrt{f(\xi) t+\left|f^{\prime}(\xi)\right| t^{2} / 2}} d t \\
& \leq C \delta(-\log f(\xi)+D) .
\end{aligned}
$$

We next calculate $Z_{2}$. We have

$$
Z_{2}=\int_{\delta}^{\xi} \frac{1}{\sqrt{f(\xi) t+\left|f^{\prime}(\xi)\right| t^{2} / 2}} d t \leq C \int_{\delta}^{\xi} \frac{1}{t} d t \leq-C \log \delta .
$$


By (40)-(44), (46), (47), and Taylor expansion, we obtain

$$
\begin{aligned}
\sqrt{\lambda}= & \frac{1}{\sqrt{\left|f^{\prime}(\xi)\right|}}(-\log f(\xi)+D)(1+O(\delta))+O(-\log \delta) \\
= & \frac{1}{\sqrt{\left|f^{\prime}(a)\right|}}(1+O(a-\xi))(1+O(\delta)) \\
& \times(-\log (a-\xi)+O(\log \delta))+O(\log \delta) \\
= & \frac{1}{\sqrt{\left|f^{\prime}(a)\right|}}(-\log (a-\xi)+O(\delta \log (a-\xi))+O(\log \delta)) \\
= & \frac{1}{\sqrt{\left|f^{\prime}(a)\right|}}(-\log (a-\xi)+O(\log \delta)) .
\end{aligned}
$$

This implies (15). Thus, the proof is complete.

\section{Proof of Theorem 3}

In this section, let $\xi \rightarrow \theta_{0}$ with $\theta_{0}<\xi$. We write $\delta:=\delta_{\xi}$ defined in the statement in Theorem 3. By (38), we have

$$
\begin{aligned}
\sqrt{\lambda}= & \frac{1}{\sqrt{2}} \int_{0}^{\xi} \frac{1}{\sqrt{F(\xi)-F(\theta)}} d \theta \\
= & \frac{1}{\sqrt{2}}\left(\int_{0}^{a} \frac{1}{\sqrt{F(\xi)-F(\theta)}} d \theta+\int_{a}^{\theta_{0}} \frac{1}{\sqrt{F(\xi)-F(\theta)}} d \theta\right. \\
& \left.\quad+\int_{\theta_{0}}^{\xi} \frac{1}{\sqrt{F(\xi)-F(\theta)}} d \theta\right) \\
:= & \frac{1}{\sqrt{2}}(I+I I+I I I) .
\end{aligned}
$$

Lemma 8. As $\xi \rightarrow \theta_{0}$,

$$
I=\frac{\sqrt{2}}{\sqrt{\left|f^{\prime}(a)\right|}}\left(-\frac{1}{2} \log \left(\xi-\theta_{0}\right)+O(\log \delta)\right) .
$$

Proof. We have

$$
\begin{aligned}
I= & \int_{a-\delta}^{a} \frac{1}{\sqrt{F(\xi)-F(\theta)}} d \theta \\
& +\int_{0}^{a-\delta} \frac{1}{\sqrt{F(\xi)-F(\theta)}} d \theta:=W_{1}+W_{2} .
\end{aligned}
$$

We first calculate $W_{1}$. For $a-\delta<\theta<a$, by (14) and Taylor expansion, we have

$$
\begin{aligned}
F(\xi) & -F(\theta) \\
= & \int_{\theta}^{\xi} f(s) d s=\int_{a}^{\theta_{0}} f(s) d s \\
& +\int_{\theta_{0}}^{\xi} f(s) d s+\int_{\theta}^{a} f(s) d s=\int_{\theta_{0}}^{\xi} f(s) d s \\
& +\int_{\theta}^{a} f(s) d s=f\left(\theta_{0}\right)\left(\xi-\theta_{0}\right)\left(1+O\left(\xi-\theta_{0}\right)\right) \\
& +\frac{1}{2}\left|f^{\prime}(a)\right|(1+O(\delta))(a-\theta)^{2} .
\end{aligned}
$$

By (51) and (52), we obtain

$$
\begin{aligned}
& W_{1}=\int_{a-\delta}^{a}\left(f\left(\theta_{0}\right)\left(\xi-\theta_{0}\right)\left(1+O\left(\xi-\theta_{0}\right)\right)\right. \\
& \left.\quad+\frac{\left|f^{\prime}(a)\right|(1+O(\delta))(a-\theta)^{2}}{2}\right)^{-1 / 2} d \theta \\
& =\int_{0}^{\delta}\left(f\left(\theta_{0}\right)\left(\xi-\theta_{0}\right)\left(1+O\left(\xi-\theta_{0}\right)\right)\right. \\
& \left.\quad+\frac{\left|f^{\prime}(a)\right|(1+O(\delta)) y^{2}}{2}\right)^{-1 / 2} d y \\
& :=\int_{0}^{\delta} \frac{1}{\sqrt{D_{1}+D_{2} y^{2}}} d y,
\end{aligned}
$$

where

$$
\begin{aligned}
& D_{1}:=f\left(\theta_{0}\right)\left(\xi-\theta_{0}\right)\left(1+O\left(\xi-\theta_{0}\right)\right), \\
& D_{2}:=\frac{1}{2}\left|f^{\prime}(a)\right|(1+O(\delta)) .
\end{aligned}
$$

Then we obtain

$W_{1}$

$$
\begin{aligned}
& =\left[\frac{1}{\sqrt{D_{2}}} \log \left|2 D_{2} y+2 \sqrt{D_{2}\left(D_{2} y^{2}+D_{1}\right)}\right|\right]_{0}^{\delta} \\
& =\frac{1}{\sqrt{D_{2}}}\left(\log \left|2 D_{2} \delta+2 \sqrt{D_{2}\left(D_{2} \delta^{2}+D_{1}\right)}\right|-\log 2 \sqrt{D_{1} D_{2}}\right) \\
& =\frac{1}{\sqrt{D_{2}}}\left(-\frac{1}{2} \log \left(\xi-\theta_{0}\right)+O(\log \delta)\right)
\end{aligned}
$$




$$
\begin{aligned}
& =\frac{\sqrt{2}}{\sqrt{\left|f^{\prime}(a)\right|}}(1+O(\delta))\left(-\frac{1}{2} \log \left(\xi-\theta_{0}\right)+O(\log \delta)\right) \\
& =\frac{\sqrt{2}}{\sqrt{\left|f^{\prime}(a)\right|}}\left(-\frac{1}{2} \log \left(\xi-\theta_{0}\right)+O(\log \delta)\right) .
\end{aligned}
$$

We next calculate $W_{2}$. For $0<\theta<a-\delta$, by (A.2) and (14), we have

$$
\begin{aligned}
F(\xi)-F(\theta) & =\int_{\theta}^{\xi} f(s) d s \\
& =\int_{\theta}^{a} f(s) d s+\int_{a}^{\xi} f(s) d s \\
& \geq \int_{\theta}^{a} f(s) d s \geq C\left|f^{\prime}(a)\right|(a-\theta) .
\end{aligned}
$$

By this, we obtain

$$
\left|W_{2}\right| \leq \int_{0}^{a-\delta} \frac{1}{\sqrt{\left|f^{\prime}(a)\right|(a-\theta)}} d s \leq C .
$$

This along with (55) implies (50). Thus the proof is complete.

Lemma 9. As $\xi \rightarrow \theta_{0}$,

$$
I I=\frac{\sqrt{2}}{\sqrt{\left|f^{\prime}(a)\right|}}\left(-\frac{1}{2} \log \left(\xi-\theta_{0}\right)+O(\log \delta)\right) .
$$

Proof. We have

$$
\begin{aligned}
I I= & \int_{a}^{\theta_{0}} \frac{1}{\sqrt{F(\xi)-F(\theta)}} d \theta \\
= & \int_{a}^{a+\delta} \frac{1}{\sqrt{F(\xi)-F(\theta)}} d \theta \\
& +\int_{a+\delta}^{\theta_{0}} \frac{1}{\sqrt{F(\xi)-F(\theta)}} d \theta:=V_{1}+V_{2} .
\end{aligned}
$$

We first calculate $V_{1}$. By (14) and Taylor expansion, for $a<$ $\theta<a+\delta$, we obtain

$$
\begin{aligned}
F(\xi) & -F(\theta) \\
= & \int_{\theta}^{\xi} f(s) d s=\int_{\theta_{0}}^{\xi} f(s) d s+\int_{a}^{\theta_{0}} f(s) d s \\
& -\int_{a}^{\theta} f(s) d s=f\left(\theta_{0}\right)\left(\xi-\theta_{0}\right)\left(1+O\left(\xi-\theta_{0}\right)\right) \\
& +\frac{1}{2}\left|f^{\prime}(a)\right|(1+O(\delta))(a-\theta)^{2}
\end{aligned}
$$

By this and (55), we obtain

$$
\begin{aligned}
V_{1} & =\int_{a}^{a+\delta} \frac{1}{\sqrt{F(\xi)-F(\theta)}} d \theta \\
& =W_{1}=\frac{1}{\sqrt{\left|f^{\prime}(a)\right|}}\left(-\frac{1}{2} \log \left(\xi-\theta_{0}\right)+O(\log \delta)\right) .
\end{aligned}
$$

We next calculate $V_{2}$. Let an arbitrary $0<\epsilon \ll 1$ be fixed. Then,

$$
\begin{aligned}
V_{2}= & \int_{a+\delta}^{\theta_{0}} \frac{1}{\sqrt{F(\xi)-F(\theta)}} d \theta \\
= & \int_{\theta_{0}-\epsilon}^{\theta_{0}} \frac{1}{\sqrt{F(\xi)-F(\theta)}} d \theta \\
& +\int_{a+\delta}^{a+\epsilon} \frac{1}{\sqrt{F(\xi)-F(\theta)}} d \theta \\
& +\int_{a+\epsilon}^{\theta_{0}-\epsilon} \frac{1}{\sqrt{F(\xi)-F(\theta)}} d \theta:=V_{21}+V_{22}+V_{23} .
\end{aligned}
$$

We calculate $V_{21}$. For $\theta_{0}-\epsilon<\theta<\theta_{0}$, we obtain

$$
\begin{aligned}
F(\xi)-F(\theta) & =\int_{\theta}^{\xi} f(s) d s \\
& =\int_{\theta_{0}}^{\xi} f(s) d s+\int_{\theta}^{\theta_{0}} f(s) d s \\
& \geq \int_{\theta}^{\theta_{0}} f(s) d s \geq \operatorname{Cf}\left(\theta_{0}\right)\left(\theta_{0}-\theta\right) .
\end{aligned}
$$

By this,

$$
V_{21} \leq \int_{\theta_{0}-\epsilon}^{\theta_{0}} \frac{1}{\sqrt{C f\left(\theta_{0}\right)\left(\theta_{0}-\theta\right)}} d \theta \leq C \sqrt{\epsilon}
$$

We next calculate $V_{22}$. For $a+\delta<\theta<a+\epsilon$,

$$
\begin{aligned}
F(\xi)-F(\theta) & =\int_{a}^{\xi} f(s) d s-\int_{a}^{\theta} f(s) d s \\
& \geq-\int_{a}^{\theta} f(s) d s \geq C\left|f^{\prime}(a)\right|(\theta-a) .
\end{aligned}
$$

By this, we obtain

$$
V_{22} \leq C \int_{a+\delta}^{a+\epsilon} \frac{1}{\sqrt{\theta-a}} d \theta \leq C \sqrt{\epsilon} .
$$

Finally, we calculate $V_{23}$. For $a+\epsilon<\theta<\theta_{0}-\epsilon$, by (14), we obtain

$$
\begin{aligned}
F(\xi)-F(\theta)= & \int_{\theta_{0}}^{\xi} f(s) d s \\
& +\int_{\theta}^{\theta_{0}} f(s) d s \geq \int_{\theta}^{\theta_{0}} f(s) d s \geq C .
\end{aligned}
$$


By this, we obtain

$$
V_{23} \leq \int_{a+\epsilon}^{\theta_{0}-\epsilon} \frac{1}{\sqrt{F(\xi)-F(\theta)}} d \theta \leq C
$$

By (62), (64), (66), and (68), we obtain

$$
V_{2} \leq C
$$

By this, (59), and (61), we obtain (58). Thus, the proof is complete.

Lemma 10. As $\xi \rightarrow \theta_{0}$,

$$
I I I=O\left(\left(\xi-\theta_{0}\right)^{1 / 2}\right)
$$

Proof. By (A.3) and (14), we know that $\tau<\theta_{0}<\xi$. Then for $\theta_{0}<\theta<\xi$, we have

$$
F(\xi)-F(\theta)=\int_{\theta}^{\xi} f(s) d s \geq C f\left(\theta_{0}\right)(\xi-\theta)
$$

By this, for $\lambda \gg 1$, we obtain

$$
I I I \leq C \int_{\theta_{0}}^{\xi} \frac{1}{\sqrt{f\left(\theta_{0}\right)(\xi-\theta)}} d \theta=\frac{2}{\sqrt{f\left(\theta_{0}\right)}} \sqrt{\xi-\theta_{0}} .
$$

Thus, the proof is complete.

Proof of Theorem 3(i). Theorem 3(i) follows directly from Lemmas $8-10$. Thus, the proof is complete.

Proof of Theorem 3(ii). By (37) and (49), we have

$$
\begin{aligned}
t_{a, \lambda} & =\int_{0}^{t_{a, \lambda}} \frac{-u_{2}^{\prime}(\lambda, t)}{\sqrt{2 \lambda\left(F(\xi)-F\left(u_{2}(\lambda, t)\right)\right)}} d t \\
& =\frac{1}{\sqrt{2 \lambda}} \int_{a}^{\xi} \frac{1}{\sqrt{F(\xi)-F(\theta)}} d \theta \\
& =\frac{1}{\sqrt{2 \lambda}}\left(\int_{a}^{\theta_{0}} \frac{1}{\sqrt{F(\xi)-F(\theta)}} d \theta+\int_{\theta_{0}}^{\xi} \frac{1}{\sqrt{F(\xi)-F(\theta)}} d \theta\right) \\
& :=\frac{1}{\sqrt{2 \lambda}}(I I+I I I) .
\end{aligned}
$$

By this, Lemmas 8-10, and direct calculation, we obtain Theorem 3(ii). Thus, the proof is complete.
Proof of Theorem 3(iii). By (37) and (49), we have

$$
\begin{aligned}
& t_{d, \lambda} \\
& =\frac{1}{\sqrt{2 \lambda}} \int_{d}^{\xi} \frac{1}{\sqrt{F(\xi)-F(\theta)}} d \theta \\
& =\frac{1}{\sqrt{2 \lambda}}\left\{\int_{d}^{\theta_{0}} \frac{1}{\sqrt{F(\xi)-F(\theta)}} d \theta+\int_{\theta_{0}}^{\xi} \frac{1}{\sqrt{F(\xi)-F(\theta)}} d \theta\right\} \\
& =\frac{1}{\sqrt{2 \lambda}}\left\{\int_{d}^{\theta_{0}} \frac{1}{\sqrt{F\left(\theta_{0}\right)-F(\theta)}} d \theta+\int_{\theta_{0}}^{\xi} \frac{1}{\sqrt{F(\xi)-F(\theta)}} d \theta\right. \\
& \left.\quad+\int_{d}^{\theta_{0}}\left(\frac{1}{\sqrt{F(\xi)-F(\theta)}}-\frac{1}{\sqrt{F\left(\theta_{0}\right)-F(\theta)}}\right) d \theta\right\} \\
& :=\frac{1}{\sqrt{2 \lambda}}\left(C_{d}+I I I+J\right) .
\end{aligned}
$$

We have only to calculate $J$. We fix a constant $\epsilon>0$ sufficiently small. Then we have

$$
\begin{aligned}
& J:=J_{1}+J_{2} \\
& =\int_{\theta_{0}-\epsilon}^{\theta_{0}}\left(F\left(\theta_{0}\right)-F(\xi)\right) \\
& \quad \times\left(\sqrt{F(\xi)-F(\theta)} \sqrt{F\left(\theta_{0}\right)-F(\theta)}\right. \\
& \left.\quad \times\left(\sqrt{F(\xi)-F(\theta)}+\sqrt{F\left(\theta_{0}\right)-F(\theta)}\right)\right)^{-1} d \theta \\
& +\int_{d}^{\theta_{0}-\epsilon}\left(F\left(\theta_{0}\right)-F(\xi)\right) \\
& \quad \times\left(\sqrt{F(\xi)-F(\theta)} \sqrt{F\left(\theta_{0}\right)-F(\theta)}\right. \\
& \left.\quad \times\left(\sqrt{F(\xi)-F(\theta)}+\sqrt{F\left(\theta_{0}\right)-F(\theta)}\right)\right)^{-1} d \theta .
\end{aligned}
$$

By (A.2), for $\theta_{0}<\xi$, we have

$$
0<F(\xi)-F\left(\theta_{0}\right) \leq f\left(\theta_{0}\right)\left(\xi-\theta_{0}\right) .
$$

Further, since $d<\theta<\theta_{0}-\epsilon<\theta_{0}<\xi$, we have

$$
F(\xi)-F(\theta) \geq C, \quad F\left(\theta_{0}\right)-F(\theta) \geq C .
$$

By this and (76), we obtain

$$
\left|J_{2}\right| \leq C\left(\xi-\theta_{0}\right) .
$$

We next calculate $J_{1}$. For $\theta_{0}-\epsilon<\theta<\theta_{0}$, we obtain

$$
\begin{gathered}
F(\xi)-F(\theta) \geq f\left(\theta_{0}\right)(\xi-\theta), \\
F\left(\theta_{0}\right)-F(\theta) \geq f\left(\theta_{0}\right)\left(\theta_{0}-\theta\right) .
\end{gathered}
$$


By (76) and (79), we obtain

$$
\begin{aligned}
\left|J_{1}\right| & \leq \int_{\theta_{0}-\epsilon}^{\theta_{0}} \frac{F(\xi)-F\left(\theta_{0}\right)}{(F(\xi)-F(\theta)) \sqrt{F\left(\theta_{0}\right)-F(\theta)}} d \theta \\
& \leq C\left(\xi-\theta_{0}\right) \int_{\theta_{0}-\epsilon}^{\theta_{0}} \frac{1}{(\xi-\theta) \sqrt{\theta_{0}-\theta}} d \theta \\
& =C\left(\xi-\theta_{0}\right) \int_{0}^{\epsilon} \frac{1}{\left(\xi-\theta_{0}+t\right) \sqrt{t}} d t \\
& \leq C\left(\xi-\theta_{0}\right) \int_{0}^{\sqrt{\epsilon}} \frac{1}{x^{2}+\left(\xi-\theta_{0}\right)} d x \\
& =C \sqrt{\xi-\theta_{0}} \tan ^{-1} \sqrt{\frac{\epsilon}{\xi-\theta_{0}}} \leq C \sqrt{\xi-\theta_{0}} .
\end{aligned}
$$

By Lemma 10, (74), (75), (78), and (80), we obtain Theorem 3(iii). Thus, the proof is complete.

\section{Proof of Theorem 4}

Let $\xi<c$ and let $\xi \rightarrow c$ in this section. The proof of Theorem 4 is similar to that of Theorem 2 . Let $\delta:=\delta_{\xi}$ defined in the statement of Theorem 4. By (38), we have

$$
\begin{aligned}
\sqrt{\lambda} & =\frac{1}{\sqrt{2}} \int_{0}^{\xi} \frac{1}{\sqrt{F(\xi)-F(\theta)}} d \theta \\
& =\frac{1}{\sqrt{2}}\left\{\int_{\xi-\delta}^{\xi} \frac{1}{\sqrt{F(\xi)-F(\theta)}} d \theta+\int_{0}^{\xi-\delta} \frac{1}{\sqrt{F(\xi)-F(\theta)}} d \theta\right\} \\
& :=\frac{1}{\sqrt{2}}\left(K_{1}+K_{2}\right) .
\end{aligned}
$$

By the same argument as that to obtain $Z_{1}$ in (41), if $\xi$ is close to $c$, we obtain

$$
K_{1}=\sqrt{\frac{2}{\left|f^{\prime}(\xi)\right|}}(-\log f(\xi)+O(\log \delta))(1+O(\delta)) .
$$

Now, we calculate $K_{2}$. We have

$$
\begin{aligned}
K_{2}= & \frac{1}{\sqrt{2}}\left(\int_{0}^{a} \frac{1}{\sqrt{F(\xi)-F(\theta)}} d \theta+\int_{a}^{\theta_{0}} \frac{1}{\sqrt{F(\xi)-F(\theta)}} d \theta\right. \\
& \left.\quad+\int_{\theta_{0}}^{\xi-\delta} \frac{1}{\sqrt{F(\xi)-F(\theta)}} d \theta\right) \\
:= & \frac{1}{\sqrt{2}}\left(K_{21}+K_{22}+K_{23}\right) .
\end{aligned}
$$

We calculate $K_{21}$. Since $\xi \rightarrow c$, by (4) and (5), for $0<\theta<a$, we have $F(\xi)-F(\theta) \geq C$. By this, we obtain

$$
K_{21} \leq \int_{0}^{a} \frac{1}{\sqrt{C}} d s \leq C .
$$

We calculate $K_{22}$. Since $\xi \rightarrow c$, by (4), for $a<\theta<\theta_{0}$, we have

$$
\begin{aligned}
F(\xi)-F(\theta) & =\int_{\theta}^{\xi} f(s) d s \\
& =\int_{\theta}^{\theta_{0}} f(s) d s+\int_{\theta_{0}}^{\xi} f(s) d s \\
& \geq \int_{\theta_{0}}^{\xi} f(s) d s \geq C .
\end{aligned}
$$

This implies that $K_{22} \leq C$. Finally, we calculate $K_{23}$. Let $\theta_{0}<$ $\theta<\xi-\delta$. By (6), we have $\left|f^{\prime}(\theta)\right|<\left|f^{\prime}(y)\right|<\left|f^{\prime}(c)\right|$ for $\theta<y<c$. By this and Taylor expansion, we obtain

$$
\begin{aligned}
F(\xi) & -F(\theta) \\
& =f(\xi)(\xi-\theta)+\frac{1}{2}\left|f^{\prime}\left(\xi_{\theta}\right)\right|(\xi-\theta)^{2} \geq C(\xi-\theta)^{2},
\end{aligned}
$$

where $\theta<\xi_{\theta}<\xi$. By this, we obtain

$$
\begin{aligned}
K_{23} & \leq \int_{\theta_{0}}^{\xi-\delta} \frac{1}{\sqrt{C(\xi-\theta)^{2}}} d \theta \\
& \leq \int_{\theta_{0}}^{\xi-\delta} \frac{1}{\xi-\theta} d \theta \leq C(-\log \delta) .
\end{aligned}
$$

By (81), (82), (83), (87), and the same calculation as that in (48), we obtain Theorem 4 . Thus, the proof is complete.

\section{Proofs of Theorems 5 and 6}

We first characterize $\xi=\xi_{0}$. Let $\lambda(\xi)$ be the curve consisting of $\lambda_{2}(\xi)$ and $\lambda_{3}(\xi)$. We know that $\xi_{0}$ is determined by $\lambda^{\prime}\left(\xi_{0}\right)=0$. By this and (38), we obtain

$$
\begin{aligned}
(\sqrt{\lambda(\xi)})_{\xi}= & \frac{1}{\sqrt{2}} \int_{0}^{1} \frac{1}{\sqrt{F(\xi)-F(\xi s)}} d s \\
& -\frac{\xi}{2 \sqrt{2}} \int_{0}^{1} \frac{f(\xi)-s f(\xi s)}{(F(\xi)-F(\xi s))^{3 / 2}} d s
\end{aligned}
$$

By this, we see that $0<\xi_{0}<1$ satisfies the following equation:

$$
2 \int_{0}^{1} \frac{1}{\sqrt{F\left(\xi_{0}\right)-F\left(\xi_{0} s\right)}} d s=\xi_{0} \int_{0}^{1} \frac{f(\xi)-s f(\xi s)}{(F(\xi)-F(\xi s))^{3 / 2}} d s .
$$

Proof of Theorem 6. We study the asymptotic behavior of $\lambda_{j}(\xi)(j=2,3)$ as $\xi \rightarrow \xi_{0}$. We put $\eta=\xi-\xi_{0}$ and consider the case where $|\eta| \ll 1$. For $0<s<1$, we put

$$
G(\xi, s):=F(\xi)-F(\xi s)-\left(F\left(\xi_{0}\right)-F\left(\xi_{0} s\right)\right) .
$$


Then,

$$
\begin{aligned}
F(\xi) & -F\left(\xi_{s}\right) \\
& =F\left(\xi_{0}\right)-F\left(\xi_{0} s\right)+G(\xi, s) \\
& =\left(F\left(\xi_{0}\right)-F\left(\xi_{0} s\right)\right)\left(1+\frac{G(\xi, s)}{F\left(\xi_{0}\right)-F\left(\xi_{0} s\right)}\right) \\
& :=\left(F\left(\xi_{0}\right)-F\left(\xi_{0} s\right)\right)(1+H(\xi, s) \eta) .
\end{aligned}
$$

We show that, for $0<s<1$ and $\eta$ sufficiently small,

$$
|H(\xi, s)| \leq C .
$$

Let an arbitrary $0<\epsilon \ll 1$ be fixed. First, we consider the case $1-\epsilon<s<1$. By (A.2), we have

$$
\left|F\left(\xi_{0}\right)-F\left(\xi_{0} s\right)\right|=\left|\int_{\xi_{0} s}^{\xi_{0}} f(s) d s\right| \geq f\left(\xi_{0}\right) \xi_{0}(1-s)
$$

We put $K(\xi, s)=F(\xi)-F(\xi s)$. By mean value theorem,

$$
\begin{aligned}
|G(\xi, s)| & =\left|K(\xi, s)-K\left(\xi_{0}, s\right)\right| \\
& \leq\left|\left(f\left(\widetilde{\xi}_{1}\right)-s f\left(\widetilde{\xi}_{1} s\right)\right) \eta\right| \\
& \leq\left|\left(f\left(\widetilde{\xi}_{1}\right)-f\left(\widetilde{\xi}_{1} s\right)\right) \eta\right|+\left|f\left(\widetilde{\xi}_{1} s\right) \eta(1-s)\right| \\
& \leq\left|\widetilde{\xi}_{1} f^{\prime}\left(\widetilde{\xi}_{2}\right) \eta(1-s)\right|+\left|f\left(\widetilde{\xi}_{1} s\right) \eta(1-s)\right|,
\end{aligned}
$$

where $\xi_{0} \sim \widetilde{\xi}_{1} \sim \widetilde{\xi}_{2} \sim \xi$. By this and (93), we obtain (92). Next, let $0<s \leq 1-\epsilon$. Since $\xi_{0}>\theta_{0}$ (cf. Figure 1), we have

$$
\left|F\left(\xi_{0}\right)-F\left(\xi_{0} s\right)\right| \geq C_{\epsilon}>0 .
$$

Furthermore,

$$
|G(\xi, s)| \leq\left|F(\xi)-F\left(\xi_{0}\right)\right|+\left|F(\xi s)-F\left(\xi_{0} s\right)\right| \leq C|\eta| .
$$

By this and (95), we obtain (92). For $|x| \ll 1$, we know that

$$
(1+x)^{-1 / 2}=1+\sum_{k=1}^{\infty} \frac{(2 k-1) ! !}{k ! 2^{k}}(-1)^{k} x^{k},
$$

where $(2 k-1) ! !=(2 k-1)(2 k-3) \cdots 3 \cdot 1$ for $k \geq 1$. By this, (38), and (91), for $j=2,3$, we obtain

$$
\begin{aligned}
& \sqrt{\lambda_{j}(\xi)} \\
& =\frac{\xi}{\sqrt{2}} \int_{0}^{1} \frac{1}{\sqrt{F(\xi)-F(\xi s)}} d s \\
& =\frac{\xi}{\sqrt{2}} \int_{0}^{1} \frac{1}{\sqrt{F\left(\xi_{0}\right)-F\left(\xi_{0} s\right)}}(1+H(\xi, s) \eta)^{-1 / 2} d s \\
& =\frac{\xi}{\sqrt{2}} \int_{0}^{1} \frac{1}{\sqrt{F\left(\xi_{0}\right)-F\left(\xi_{0} s\right)}} \\
& =\frac{\xi_{0}}{\sqrt{2}} \int_{0}^{1} \frac{1}{\sqrt{F\left(\xi_{0}\right)-F\left(\xi_{0} s\right)}}+\frac{\eta}{\sqrt{2}} \int_{0}^{1} \frac{1}{\sqrt{F\left(\xi_{0}\right)-F\left(\xi_{0} s\right)}} \\
& \left.+\frac{\xi_{0}+\eta}{\sqrt{2}} \sum_{k=1}^{\infty} \int_{0}^{1} \frac{1(2 k-1) ! !}{\sqrt{F\left(\xi_{0}\right)-F\left(2_{0} s\right)}}(-1)^{k} H(\xi, s)^{k} \eta^{k}\right) d s \\
& = \\
& \quad \sqrt{\lambda_{j}\left(\xi_{0}\right)}+\sum_{k=1}^{\infty} H_{k}(\xi) \eta^{k} . \\
& k ! 2^{k}
\end{aligned}
$$

By this, we obtain Theorem 6 . Thus, the proof is complete.

Proof of Theorem 5. We write $\lambda=\lambda_{1}(\xi)$. For $0<s<1$ and $0<\xi \ll 1$, we put

$$
D(\xi, s):=\frac{F(\xi)-F(\xi s)-\xi f(\xi)(1-s)}{\xi^{2} f(\xi)(1-s)^{2}} .
$$

By Taylor expansion,

$$
F(\xi)-F(\xi s)-\xi f(\xi)(1-s) \sim-\frac{\xi^{2} f^{\prime}(0)}{2}(1-s)^{2} .
$$

By (99) and (100), for $0<s<1$ and $0<\xi \ll 1$, we obtain

$$
\begin{gathered}
\sqrt{F(\xi)-F(\xi s)}=\sqrt{\xi f(\xi)} \sqrt{1-s} \sqrt{1+D(\xi, s) \xi(1-s)}, \\
|D(\xi, s)| \leq C .
\end{gathered}
$$


By this, (97), and (98), we obtain

$$
\begin{aligned}
& \sqrt{\lambda_{1}(\xi)} \\
= & \frac{\xi}{\sqrt{2}} \int_{0}^{1} \frac{1}{\sqrt{F(\xi)-F(\xi s)}} d s \\
= & \frac{\sqrt{\xi}}{\sqrt{2 f(\xi)}} \int_{0}^{1} \frac{1}{\sqrt{1-s}}(1+D(\xi, s) \xi(1-s))^{-1 / 2} d s \\
= & \frac{\sqrt{\xi}}{\sqrt{2 f(\xi)}} \\
& \times \int_{0}^{1} \frac{1}{\sqrt{1-s}} \\
\times & \frac{\sqrt{\xi}}{\sqrt{2 f(\xi)}}\left(2+\sum_{k=1}^{\infty} \frac{(2 k-1) ! !}{k ! 2^{k}}(-1)^{k} D(\xi, s)^{k}(1-s)^{k} \xi^{k}\right) d s \\
= &
\end{aligned}
$$

where

$$
L_{k}(\xi)=\int_{0}^{1} \frac{(2 k-1) ! !}{k ! 2^{k}}(-1)^{k} D(\xi, s)^{k}(1-s)^{k-1 / 2} d s
$$

Thus, we obtain Theorem 5(i). Theorem 5(ii) is obtained by Theorem 5(i) and Taylor expansion of $f(u)$ near $u=0$. Thus, the proof is complete.

\section{Proof of Theorem 7}

Proof of Theorem $7(i)$. Let $f(u)=(u-a)(u-b)(c-u)$ with $a=a_{j}, b=b_{j}, c=c_{j}(j=1,2)$. By (26) and Theorem 2, we obtain $f^{\prime}\left(a_{1}\right)=f^{\prime}\left(a_{2}\right)$. This implies that

$$
\left(c_{1}-a_{1}\right)\left(b_{1}-a_{1}\right)=\left(c_{2}-a_{2}\right)\left(b_{2}-a_{2}\right) \text {. }
$$

By direct calculation,

$$
\begin{aligned}
F(\xi) & -F(\xi s) \\
= & -\frac{1}{4} \xi^{4}(1-s)\left(1+s+s^{2}+s^{3}\right) \\
& +\frac{1}{3}(a+b+c) \xi^{3}(1-s)\left(1+s+s^{2}\right) \\
& -\frac{1}{2}(a b+b c+c a) \xi^{2}(1-s)(1+s)+a b c \xi(1-s) .
\end{aligned}
$$

Let $A:=(a b+b c+c a) /(2 a b c)$. By using (38), (105), and the calculation in Section 5, we obtain

$$
\begin{aligned}
\sqrt{\lambda_{1}(\xi)} & =\frac{\sqrt{\xi}}{\sqrt{2 a b c}} \int_{0}^{1} \frac{1}{\sqrt{1-s}}\left(1-A(1+s) \xi+O\left(\xi^{2}\right)\right)^{-1 / 2} d s \\
& =\frac{\sqrt{\xi}}{\sqrt{2 a b c}} \int_{0}^{1} \frac{1}{\sqrt{1-s}}\left(1+\frac{1}{2} A \xi(1+s)+O\left(\xi^{2}\right)\right) d s \\
& =\frac{\sqrt{\xi}}{\sqrt{2 a b c}}\left(2+\frac{5}{3} A \xi+O\left(\xi^{2}\right)\right) .
\end{aligned}
$$

This implies that

$$
\lambda_{1}(\xi)=\frac{2}{a b c} \xi\left(1+\frac{5}{3} A \xi+O\left(\xi^{2}\right)\right) .
$$

By this, (27), (28), and Theorem 2, we obtain

$$
\begin{gathered}
a_{1} b_{1} c_{1}=a_{2} b_{2} c_{2} \\
a_{1} b_{1}+b_{1} c_{1}+c_{1} a_{1}=a_{2} b_{2}+b_{2} c_{2}+c_{2} a_{2} .
\end{gathered}
$$

By (104), (108), (109), and direct calculation, we easily obtain $a_{1}=a_{2}, b_{1}=b_{2}$, and $c_{1}=c_{2}$. Thus, the proof is complete.

Proof of Theorem 7(ii). By Theorem 2 and (28), we obtain $f^{\prime}\left(a_{1}\right)=f^{\prime}\left(a_{2}\right)$ and $f^{\prime}\left(c_{1}\right)=f^{\prime}\left(c_{2}\right)$. By this, we obtain (104) and

$$
\left(c_{1}-a_{1}\right)\left(c_{1}-b_{1}\right)=\left(c_{2}-a_{2}\right)\left(c_{2}-b_{2}\right)>0 .
$$

By this and (104), we obtain $\left(c_{1}-a_{1}\right)^{2}=\left(c_{2}-a_{2}\right)^{2}$. By this and (104), we have

$$
p:=b_{1}-a_{1}=b_{2}-a_{2}, \quad q:=c_{1}-a_{1}=c_{2}-a_{2} .
$$

By (29) and (107), we obtain (108). By (108) and (111), we obtain

$$
a_{1}\left(a_{1}+p\right)\left(a_{1}+q\right)=a_{2}\left(a_{2}+p\right)\left(a_{2}+q\right) .
$$

By this, we obtain $a_{1}=a_{2}$. By this and (111), we obtain $b_{1}=b_{2}$ and $c_{1}=c_{2}$. Thus, the proof is complete.

Proof of Theorem 7 (iii). Let $\theta_{0}:=\theta_{1,0}=\theta_{2,0}$. We put

$$
G(t):=\int_{0}^{t} y(y-p)(q-y) d y .
$$

For $j=1,2$, by (14) and (111), we obtain

$$
\begin{aligned}
0 & =\int_{a_{j}}^{\theta_{0}}\left(x-a_{j}\right)\left(x-b_{j}\right)\left(c_{j}-x\right) d x \\
& =\int_{a_{j}}^{\theta_{0}}\left(x-a_{j}\right)\left(x-\left(a_{j}+p\right)\right)\left(\left(a_{j}+q\right)-x\right) d x \\
& =\int_{0}^{\theta_{0}-a_{j}} y(y-p)(q-y) d y .
\end{aligned}
$$


We study the shape of $G(t)$ for $t \geq 0$. Since $c+a>2 b$ by (5), we have $2 p<q$. By this and direct calculation, we see that $G(0)=0$ and it decreases for $0<t<p$ and attains its minimum at $t=p$, and $G(p)<0$. Then $G(t)$ increases for $p<t<q$ and attains its maximum at $t=q$, and $G(q)=q^{3}(q-$ $2 p) / 12>0$. Then, $G(t)$ decreases for $t>q$ and $G(t) \rightarrow-\infty$ as $t \rightarrow \infty$. Therefore, there exists $p<t_{1}<q<t_{2}$ such that $G\left(t_{1}\right)=G\left(t_{2}\right)=0$. We assume that $a_{1}<a_{2}$ and we derive a contradiction. In this case, by (114), we obtain $\theta_{0}-a_{2}=t_{1}$ and $\theta_{0}-a_{1}=t_{2}$. This implies that $\theta_{0}=a_{1}+t_{2}>a_{1}+q=c_{1}$. This is a contradiction, since $b_{1}<\theta_{0}<c_{1}$. If we assume that $a_{2}<a_{1}$, we obtain the same contradiction as above. Consequently, we obtain $a_{1}=a_{2}$. By this and (111), we obtain $b_{1}=b_{2}$ and $c_{1}=c_{2}$. Thus, the proof is complete.

\section{Conflict of Interests}

The author declares that there is no conflict of interests regarding the publication of this paper.

\section{References}

[1] P. Korman, Y. Li, and T. Ouyang, "Perturbation of global solution curves for semilinear problems," Advanced Nonlinear Studies, vol. 3, no. 2, pp. 289-299, 2003.

[2] S. Cano-Casanova and J. López-Gómez, "Existence, uniqueness and blow-up rate of large solutions for a canonical class of onedimensional problems on the half-line," Journal of Differential Equations, vol. 244, no. 12, pp. 3180-3203, 2008.

[3] S. Cano-Casanova and J. López-Gómez, "Blow-up rates of radially symmetric large solutions," Journal of Mathematical Analysis and Applications, vol. 352, no. 1, pp. 166-174, 2009.

[4] R. Chiappinelli, "A-priori bounds and asymptotics on the eigenvalues in bifurcation problems for perturbed self-adjoint operators," Journal of Mathematical Analysis and Applications, vol. 354, no. 1, pp. 263-272, 2009.

[5] R. Chiappinelli, "Upper and lower bounds for higher order eigenvalues of some semilinear elliptic equations," Applied Mathematics and Computation, vol. 216, no. 12, pp. 3772-3777, 2010.

[6] R. Chiappinelli, M. Furi, and M. P. Pera, "A new theme in nonlinear analysis: continuation and bifurcation of the unit eigenvectors of a perturbed linear operator," Communications in Applied Analysis, vol. 15, no. 2, pp. 299-312, 2011.

[7] R. Chiappinelli, "Variational methods for NLEV approximation near a bifurcation point," International Journal of Mathematics and Mathematical Sciences, vol. 2012, Article ID 102489, 32 pages, 2012.

[8] J. López-Gómez, A. Tellini, and F. Zanolin, "High multiplicity and complexity of the bifurcation diagrams of large solutions for a class of superlinear indefinite problems," Communications on Pure and Applied Analysis, vol. 13, no. 1, pp. 1-73, 2014.

[9] P. Korman, Y. Li, and T. Ouyang, "Exact multiplicity results for boundary value problems with nonlinearities generalising cubic," Proceedings of the Royal Society of Edinburgh A, vol. 126, no. 3, pp. 599-616, 1996.

[10] P. Korman, Y. Li, and T. Ouyang, "Computing the location and direction of bifurcation," Mathematical Research Letters, vol. 12, no. 5-6, pp. 933-944, 2005.
[11] P. Korman, "Global solution branches and exact multipli city of solutions for two point boundary value problems," in Handbook of Differential Equations: Ordinary Differential Equations, vol. 3, pp. 547-606, Elsevier/North-Holland, Amsterdam, The Netherlands, 2006.

[12] P. Korman, Global Solution Curves for Semilinear Elliptic Equations, World Scientific, Hackensack, NJ, USA, 2012.

[13] T. Shibata, "Local structure of bifurcation curves for nonlinear Sturm-Liouville problems," Journal of Mathematical Analysis and Applications, vol. 369, no. 2, pp. 583-594, 2010. 




Advances in

Operations Research

mansans



The Scientific World Journal
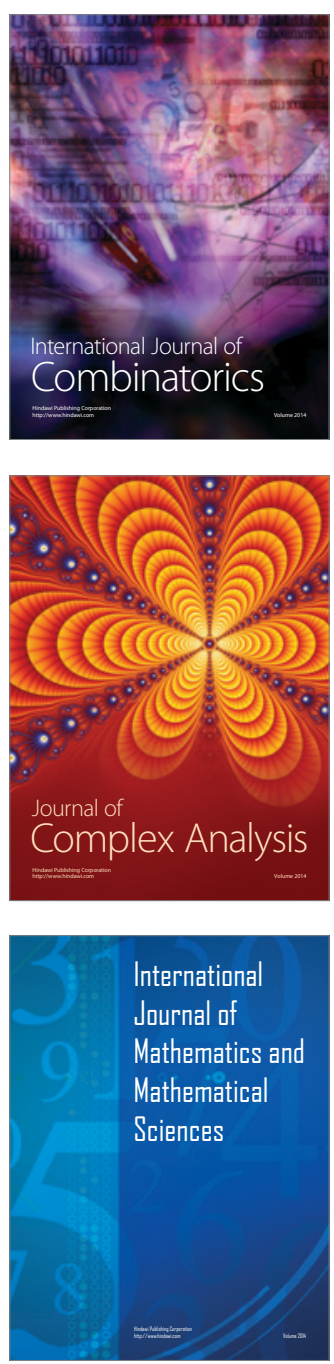
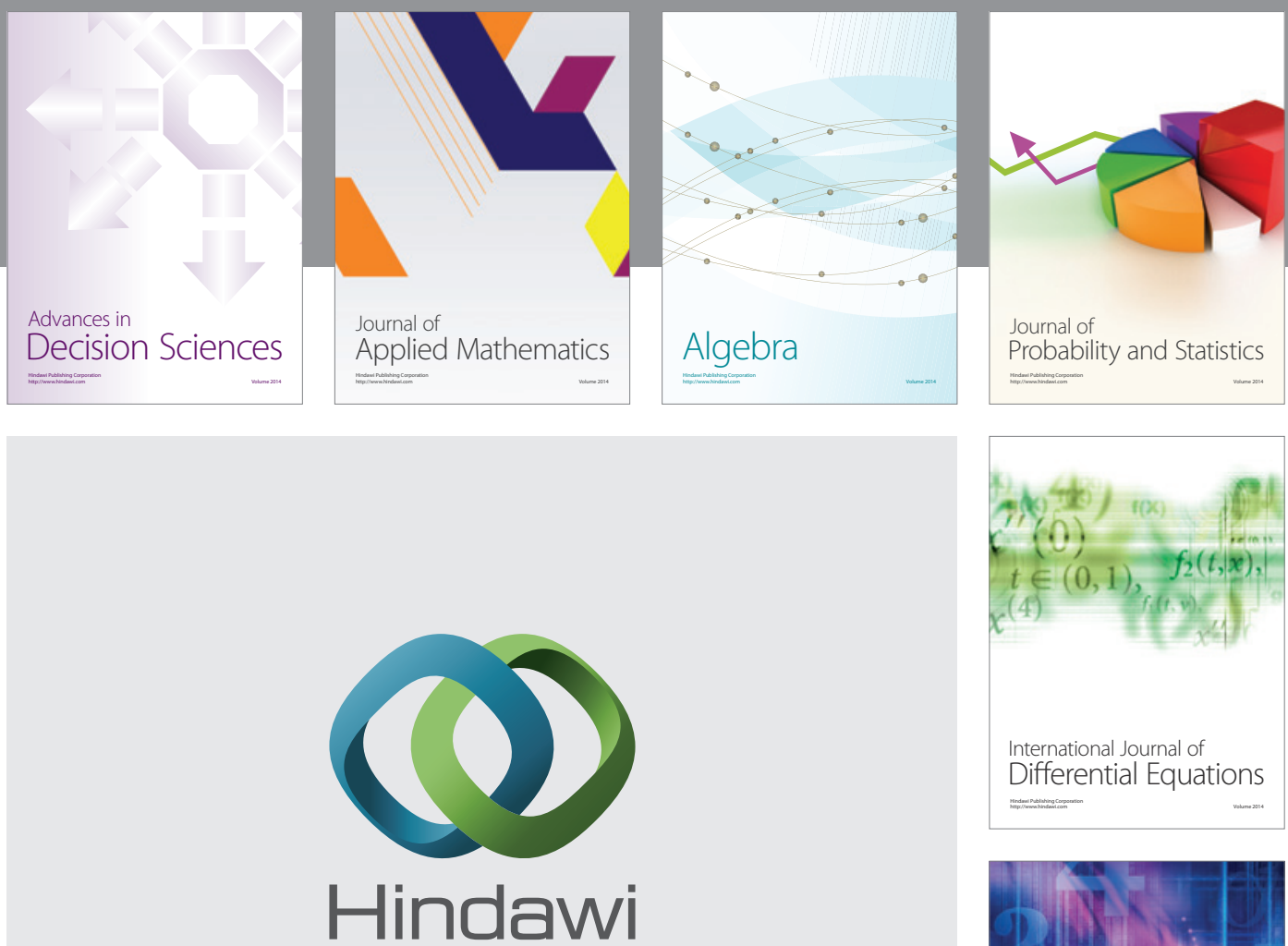

Submit your manuscripts at http://www.hindawi.com
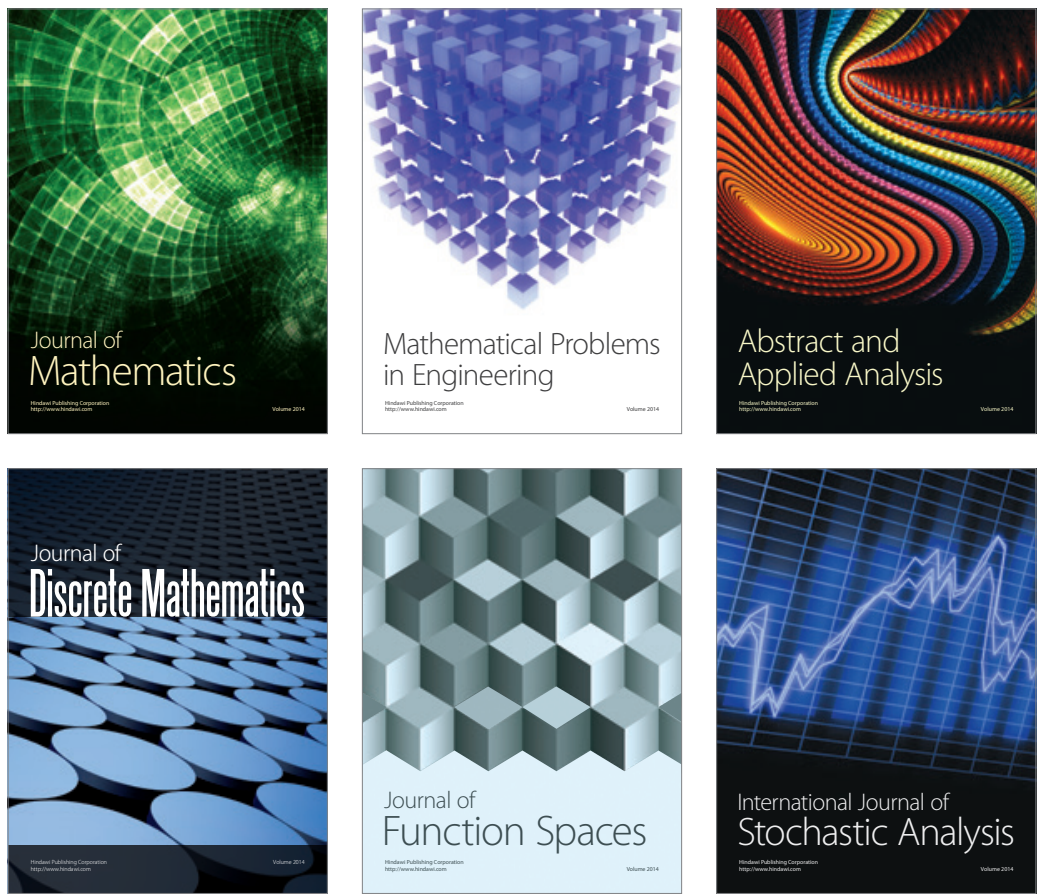

Journal of

Function Spaces

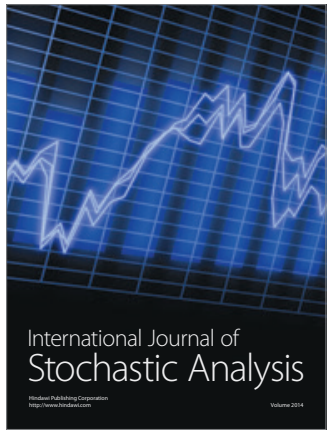

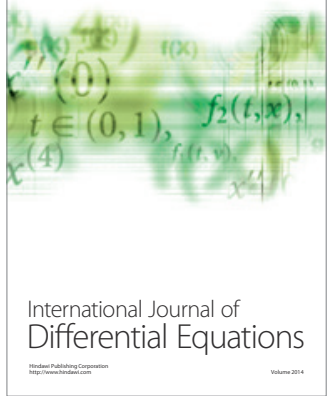
